\title{
The Rigidity of Spherical Frameworks: Swapping Blocks and Holes
}

\author{
Wendy Finbow * \\ Elissa Ross ${ }^{\dagger}$ \\ Walter Whiteley $\ddagger$
}

October 21, 2018

\begin{abstract}
A significant range of geometric structures whose rigidity is explored for both practical and theoretical purposes are formed by modifying generically isostatic triangulated spheres. In the block and hole structures $(\mathcal{P}, \boldsymbol{p})$, some edges are removed to make holes, and other edges are added to create rigid sub-structures called blocks. Previous work noted a combinatorial analogy in which blocks and holes played equivalent roles. In this paper, we connect stresses in such a structure $(\mathcal{P}, \boldsymbol{p})$ to first-order motions in a swapped structure $(\overline{\mathcal{P}}, \boldsymbol{p})$, where holes become blocks and blocks become holes. When the initial structure is geometrically isostatic, this shows that the swapped structure is also geometrically isostatic, giving the strongest possible correspondence. We use a projective geometric presentation of the statics and the motions, to make the key underlying correspondences transparent.
\end{abstract}

MSC: Primary 52C25; Secondary: 51N15 70C20 70B15

Key words: generic rigidity, static rigidity, infinitesimal rigidity, projective geometry, spherical structures, duality

*Department of Mathematics, St. Mary's University. The author was supported by NSERC (Canada) and York University

${ }^{\dagger}$ Department of Mathematics and Statistics, York University. The author was supported in part under a grant from NSERC (Canada).

${ }^{\ddagger}$ Department of Mathematics and Statistics, York University. The author was supported in part by a grant from NSERC (Canada). 

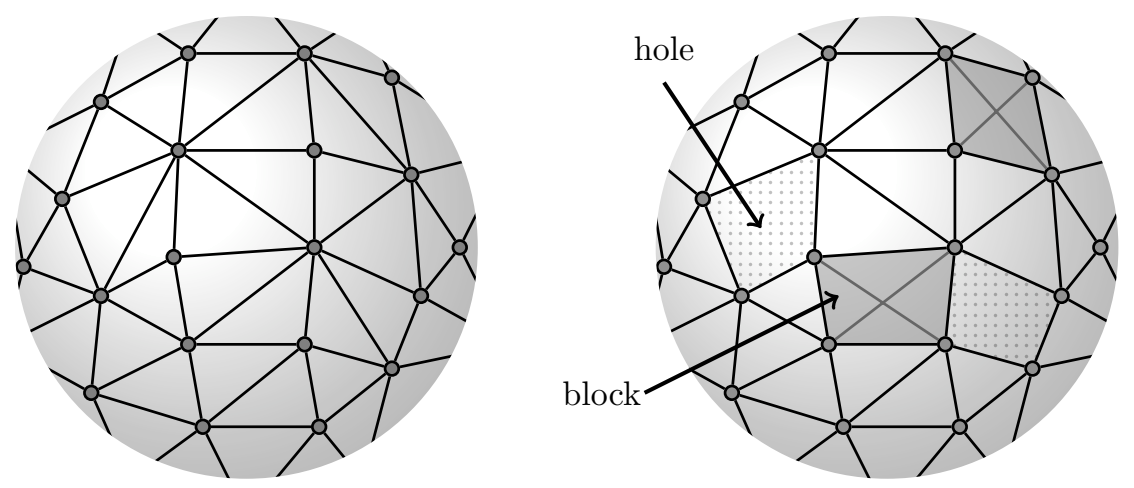

Figure 1: A triangulated sphere (left). Removing some edges creates holes (dotted), and replacing the edges elsewhere creates blocks (shaded).

\section{Introduction}

The general problem of which graphs can be realized in 3-space as an isostatic (rigid and independent) bar and joint framework is a major unsolved problem in rigidity theory [8, 15]. In the absence of a general characterization, it becomes significant to investigate certain classes of graphs and to confirm the rigidity of almost all realizations of the graphs in 3-space (generic rigidity).

Historically, from the work of Cauchy and Dehn, [2, 4], we know that convex triangulated spheres are isostatic, and therefore, any generic realization of the corresponding 3-connected planar graphs with $|E|=3|V|-6$ is also isostatic [6]. In a previous paper, [5], two of the authors considered the following process: remove some edges of a convex triangulated sphere (creating holes), insert the same number of edges to create isostatic subpieces (blocks) in the sphere, and leave the 'untouched' portions as triangulated surfaces. The authors verified that under certain conditions this process preserves the generically isostatic (rigid, independent) nature of such block and hole polyhedra.

A read through the paper [5] suggests that the blocks and holes play 'dual' roles. That is, if we look at the necessary conditions and the conjectures for isostatic block and hole frameworks in that paper, we can swap the faces which were blocks into holes and faces which were holes into blocks. This suggests that if a block and hole framework is generically isostatic, then the swapped framework is also generically isostatic. 
In this paper, we show a stronger set of geometric results that have these observed combinatorial connections as corollaries. Let $\mathcal{P}$ be a block and hole polyhedron, and let $\overline{\mathcal{P}}$ be the swapped polyhedron (blocks and holes interchanged). Let $G(\mathcal{P})$ and $G(\overline{\mathcal{P}})$ represent the graphs of the polyhedron and swapped polyhedron respectively. Let $\boldsymbol{p}$ be an embedding of the graph into $\mathbb{R}^{3}$, and let $G(\mathcal{P}, \boldsymbol{p})$ and $G(\overline{\mathcal{P}}, \boldsymbol{p})$ be the embedded frameworks of $\mathcal{P}$ and $\overline{\mathcal{P}}$ respectively. We show that:

1. if a block and hole polyhedral framework $G(\mathcal{P}, \boldsymbol{p})$ has a non-trivial first-order motion, then the swapped block and hole structure $G(\overline{\mathcal{P}}, \boldsymbol{p})$ has a static self-stress in the same configuration; and

2. if a block and hole polyhedral framework $G(\mathcal{P}, \boldsymbol{p})$ has a static selfstress, then the swapped block and hole structure $G(\overline{\mathcal{P}}, \boldsymbol{p})$ has a nontrivial first-order motion in the same configuration.

From these basic results we conclude that a block and hole polyhedron is geometrically isostatic (rigid, independent) at a given configuration $\boldsymbol{p}$ if and only if the swapped block and hole polyhedron is also isostatic at $\boldsymbol{p}$. The generic results also follow: a block and hole polyhedron is generically isostatic if, and only if the swapped polyhedron is generically isostatic.

The methods used are an extension of previous geometric work connecting the first-order motions of spherical polyhedra formed with all faces rigid and edges as hinges, and the stresses on a framework, that is, the stresses on the underlying graph of vertices and edges of the polyhedron [3. If the entire underlying polyhedron has only triangular faces, the correspondence is also implicit in earlier work on Alexandrov's Theorem [19]. Here we provide an overarching projective geometric analysis that gives a general theory extending all of these previous results.

In Section 2 we provide a formal introduction to our object of study, the block and hole polyhedron, and the basic projective geometric theory of static rigidity and infinitesimal rigidity in space. We also provide a brief introduction to the projective geometry and the language of the GrassmannCayley algebra in which we are presenting the rigidity. This projective presentation makes the correspondence much more transparent than an alternate Euclidean presentation would be. It also highlights the advantages of placing infinitesimal and static rigidity into projective geometry: both increased simplicity and increased generality.

In Section 3, we prove the main result for separated block and hole polyhedra, which is a simplified setting where we assume no pair of holes or blocks share a vertex. In section 4.1 we introduce gussets to account for 
remaining cases. Finally, in Section 5 we outline some extensions and discuss further implications of this work. In particular, it is always of interest to determine which configurations make a generically isostatic graph into a geometrically isostatic framework. We review some connections of this work with prior work on these polynomial pure conditions [13].

\section{Background}

We begin by introducing the basic combinatorial object for this study. This will be followed by a general introduction to the projective geometric theory of the statics of frameworks and the projective geometric theory of the (firstorder) motions of hinge structures. In the final subsection we will bring these three pieces together to give the notation and background for the following sections.

\subsection{Block and Hole Polyhedra}

In [5, 17, we introduced a construction process for block and hole polyhedra. This construction permitted us to extract the generic static rigidity properties of the framework of the block and hole polyhedra from the generic behaviour of an underlying 'base' block and hole polyhedron.

In this paper, a number of the construction details from [5] and [17] will not be relevant. Thus, we give a simplified definition of a block and hole polyhedra, starting with the definition of an abstract spherical polyhedron.

An abstract spherical polyhedron can be constructed from a spherical drawing of a 3-connected planar graph $G$ (no edges crossings), adding the regions created in the drawing as the 'faces' of the polyhedron. This face structure is unique, given 3-connectivity.

Definition 2.1 A block and hole polyhedron $\mathcal{P}$ with vertex set $V$, edge set $E$, and face set $\mathcal{F}$ is an abstract spherical polyhedron whose faces $\mathcal{F}=$ $\left(\mathcal{B}_{\mathcal{P}}, \mathcal{H}_{\mathcal{P}}, \mathcal{I}_{\mathcal{P}}\right)$ are partitioned into three mutually disjoint sets, $\mathcal{B}_{\mathcal{P}}, \mathcal{H}_{\mathcal{P}}$, and $\mathcal{T}_{\mathcal{P}}$. The set $\mathcal{B}_{\mathcal{P}}$ contains the faces designated as blocks and the set $\mathcal{H}_{\mathcal{P}}$ contains the faces designated as holes. The remaining faces are triangulated on their vertices, and the collection of resulting triangular faces forms the set $\mathcal{T}_{\mathcal{P}}$.

In Section 5.3, we consider the small modifications needed to include 2-connected planar graphs. 


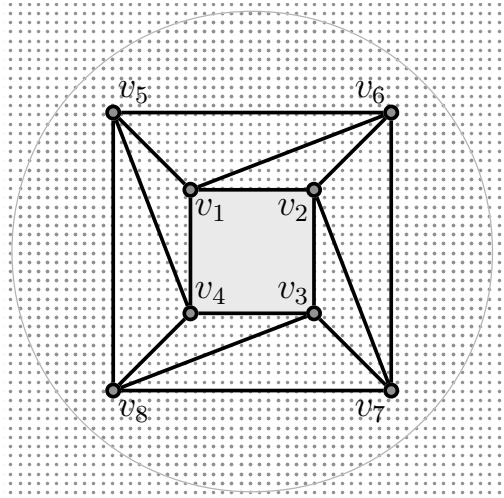

(a)

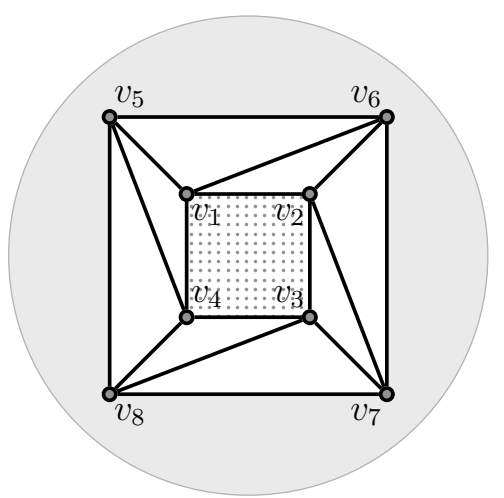

(b)

Figure 2: An example of a block and hole polyhedron $\mathcal{P}$ shown in (a). Blocks are denoted by grey faces, and holes are denoted by dotted faces. The circular region is understood to be a connected face, in this case a hole face of the polyhedron. The swapped version of this polyhedron, $\overline{\mathcal{P}}$ is shown in (b).

Central to our analysis of block and hole polyhedra is the process of 'swapping', in which holes become blocks and blocks become holes (See Figure 2p.

Definition 2.2 Given a block and hole polyhedron $\mathcal{P}$, the swapped block and hole polyhedron $\overline{\mathcal{P}}=\left(\mathcal{B}_{\overline{\mathcal{P}}}, \mathcal{H}_{\overline{\mathcal{P}}}, \mathcal{T}_{\overline{\mathcal{P}}}\right)$ is the block and hole polyhedron with blocks and holes interchanged; that is, $\mathcal{B}_{\overline{\mathcal{P}}}=\mathcal{H}_{\mathcal{P}}, \mathcal{H}_{\overline{\mathcal{P}}}=\mathcal{B}_{\mathcal{P}}$ and $\mathcal{T}_{\overline{\mathcal{P}}}=\mathcal{T}_{\mathcal{P}}$.

It is immediate that $\overline{\overline{\mathcal{P}}}=\mathcal{P}$.

Recall that each edge $\{i, j\}$ of a block and hole polyhedra $\mathcal{P}$ joins two distinct vertices $i$ and $j$ of $\mathcal{P}$, and separates two distinct faces, $F^{k}$ and $F^{m}$ of $\mathcal{P}$ (Figure 3). We introduce a pairing of the vertex-edge-vertex pairs and the face-edge-face pairs obtained from the orientation of the spherical surface, $\mathcal{P}$. We write $\left\langle v_{i}, v_{j} ; F^{k}, F^{m}>\right.$ for the edge patch with oriented edge from $i$ to $j$ and the oriented pair of faces $\left(F^{k}, F^{m}\right)$ which crosses the oriented edge after a $90^{\circ}$ counterclockwise turn. The reversed patch is $\left\langle v_{j}, v_{i} ; F^{m}, F^{k}\right\rangle$; see Figure 3. This spherical drawing of the block and hole polyhedra $\mathcal{P}$ also gives a cycle of faces and edges around each vertex. We use such cycles and patches in our later analysis. 

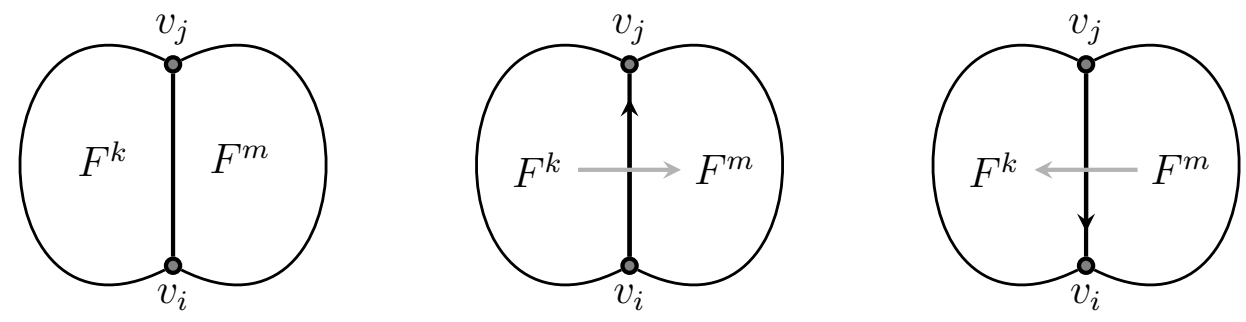

$$
<v_{i}, v_{j} ; F^{k}, F^{m}>\quad<v_{j}, v_{i} ; F^{m}, F^{k}>
$$

Figure 3: Two vertices joined by an edge and the two faces meeting at an edge form two oriented edge patches.

\subsection{Stresses of Frameworks}

In this section, we outline the basic projective theory of static rigidity for frameworks, following [3]. A bar and joint framework, $\mathcal{F}=(G, \boldsymbol{p})$ in projective 3-space is a finite graph $G=(V, E)$, where $V=\{1,2, \ldots, v\}$ is a set of vertices and $E$ is a set of unordered pairs of vertices called edges, and a mapping $\boldsymbol{p}: V \mapsto \mathbb{P}^{3}$ such that if $\{i, j\} \in E$ then $\boldsymbol{p}(i) \neq \boldsymbol{p}(j)$. If $i \in V$, we call $\boldsymbol{p}(i)=\boldsymbol{p}_{i}$ a joint of $\mathcal{F}$. We call $\left(\boldsymbol{p}_{i}, \boldsymbol{p}_{j}\right)$ a bar of the framework, and when the framework is clear we denote the bar as $(i, j)$ or $i j$. We refer to the mapping $\boldsymbol{p}$ as a configuration, to $E$ as the set of bars, and to $V$ as the set of joints of the framework.

We are particularly interested in internal stresses that occur on the bars and joints of frameworks. For ease of notation and simplicity, we do this in a projective setting. For an introduction to Grassmann-Cayley algebra and projective geometry, see [3, 11, 12].

Let $\boldsymbol{a}=\left(a_{1}, a_{2}, a_{3}, 1\right)$ and $\boldsymbol{b}=\left(b_{1}, b_{2}, b_{3}, 1\right)$ be two points in $\mathbb{P}^{3}$. The line through $\boldsymbol{a}$ and $\boldsymbol{b}$ may be represented by the Grassmann or exterior product, $\boldsymbol{a} \vee \boldsymbol{b}=\boldsymbol{a} \boldsymbol{b}$ (read a join $\mathrm{b}$ ), defined to be the six $2 \times 2$ minors $\left(d_{14}, d_{24}, d_{34}, d_{23}, d_{31}, d_{12}\right)$ of the $2 \times 4$ matrix

$$
\left[\begin{array}{llll}
a_{1} & a_{2} & a_{3} & 1 \\
b_{1} & b_{2} & b_{3} & 1
\end{array}\right]
$$

The exterior product $\boldsymbol{a b}$ is known as both a 2-extensor and the Plücker coordinates of the line through $\boldsymbol{a}$ and $\boldsymbol{b}$.

To understand the rigidity of such a framework, we examine the effect of applying forces (loads) to the structure. A force can be thought of as a 
directed segment of the line though the projective points $\mathbf{f}=\left(f_{1}, f_{2}, f_{3}, 0\right)$ and $\boldsymbol{a}=\left(a_{1}, a_{2}, a_{3}, 1\right)$, and hence the load applied to $\boldsymbol{a}$ is represented by the 2-extensor $\mathbf{F}=\mathbf{f} \boldsymbol{a}$.

We assume all forces are applied at the joints of the framework. An external load on a framework is an assignment of loads $\boldsymbol{L}=\left(\boldsymbol{L}_{1}, \boldsymbol{L}_{2}, \ldots, \boldsymbol{L}_{v}\right)$ to the joints $V=\left(\boldsymbol{p}_{1}, \boldsymbol{p}_{2}, \ldots, \boldsymbol{p}_{v}\right)$ of the framework such that each $\boldsymbol{L}_{i}$ passes through the joint $\boldsymbol{p}_{i}$. The external load is said to be in equilibrium if

$$
\sum_{i=1}^{v} \boldsymbol{L}_{i}=\mathbf{0}
$$

This set of six equations is independent if the points are not collinear, giving a vector space of equilibrium loads of dimension $3|V|-6$.

A resolution of the equilibrium load by a framework is an assignment of scalars $\lambda_{i j}$ to the bars of the framework such that for each joint $\boldsymbol{p}_{i} \in V$,

$$
\boldsymbol{L}_{i}+\sum_{\{j \mid(i, j) \in E\}} \lambda_{i j} \boldsymbol{p}_{i} \boldsymbol{p}_{j}=\mathbf{0}
$$

Definition 2.3 A framework $\mathcal{F}=(G, \boldsymbol{p})$ is statically rigid if every equilibrium load of the framework has a resolution by the bars of the framework.

The set of equations given in the vector equation (2) defines a linear transformation from the vector space of resolutions (by the bars of the framework) to the vector space of equilibrium loads.

A framework is called isostatic if its bars form a basis for the space of equilibrium loads, in other words, the framework is called isostatic if it is minimally statically rigid. It follows that in an isostatic framework, $|E|=3|V|-6$ (unless $|V| \leq 2$ ).

Throughout the remainder of this paper, we will focus on internal forces acting within the entire framework $\mathcal{F}=(G, \boldsymbol{p})$. We consider the possible tensions and compressions within the bars of a framework. Specifically, we have the bar load $\boldsymbol{B}_{i j}$ which applies equal and opposite forces at the two ends of a bar: $\boldsymbol{p}_{i} \boldsymbol{p}_{j}$ at $p_{i}$ and $\boldsymbol{p}_{j} \boldsymbol{p}_{i}$ at $p_{j}$ and 0 at all other vertices. Together, these two form an equilibrium load, and any multiple $\lambda_{i j} \boldsymbol{B}_{i j}$ is a tension $\left(\lambda_{i j}<0\right)$ or compression $\left(\lambda_{i j}>0\right)$ in the bar. The subspace generated by these internal bar forces is the space $\mathcal{B}$ of resolved loads.

We say these internal forces are in equilibrium at each joint $\boldsymbol{p}_{j}$ when $\sum_{j} \lambda_{i, j} \boldsymbol{p}_{i} \boldsymbol{p}_{j}=\mathbf{0}$ for every joint $\boldsymbol{p}_{i}$ such that $(i, j) \in E$. A self-stress on a 
bar and joint framework $\mathcal{F}$ is an assignment of scalars $\lambda_{i j}$ to the bars $(i, j)$ of the framework such that for every vertex $i$,

$$
\sum_{\{j \mid(i, j) \in E\}} \lambda_{i j}\left(\boldsymbol{p}_{i} \boldsymbol{p}_{j}\right)=\mathbf{0}
$$

A self-stress is called non-trivial if some $\lambda_{i j} \neq 0$. For simplicity, we will call a non-trivial self-stress a stress. A framework is called independent if it has only a trivial self-stress; otherwise, a framework has a non-trivial self-stress and is called dependent.

In the language of the bar loads, a self-stress is a linear dependence among the bar loads. A framework is statically rigid if $\mathcal{B}$ is the entire space of equilibrium loads. A framework is isostatic if $\mathcal{B}$ is a basis for the space of equilibrium loads.

A cut set $E^{\prime}$ of a framework is a subset of the edges of the framework (in other words, $E^{\prime} \subset E$ ) whose removal separates the framework into two or more distinct components. The following theorem gives a useful property of such cut sets.

THEOREM $2.1([3])$ Let $E^{\prime}=\left\{\left(\boldsymbol{p}_{i}, \boldsymbol{q}_{i}\right) \mid k \leq i \leq n\right\}$ be the cut set of a framework $\mathcal{F}=(G, \boldsymbol{p})$. Then for any stress $\Lambda$ on the framework,

$$
\sum_{i=k}^{n} \lambda_{i} \boldsymbol{p}_{i} \boldsymbol{q}_{i}=\mathbf{0}
$$

where $\lambda_{i} \in \Lambda$.

Theorem 2.1 says that the forces from a self-stress acting across the cut-set onto a component $C$ with edges from the cut set removed, are an equilibrium load onto this component. The overall equilibrium conditions for the larger self-stress guarantee that the coefficients of the self-stress within the component $C$ are a resolution of this cut-set load.

In our later work, we use the following isostatic substitution principle, which says, roughly speaking, that within a given framework, we can substitute an isostatic subframework attached on a subset of vertices $V^{\prime}$ for another isostatic subframework attached at $V^{\prime}$, without changing the static rigidity of the overall framework. This will follow from general principles about bases, spanning sets, and linear dependencies in vector spaces, translated into the language of the particular spaces of interest in statics: (i) the isostatic frameworks, whose bars are bases for the equilibrium loads; (ii) the 
self-stresses which are linear dependencies among the bars; and (iii) Theorem 2.1 which converts a self-stress across a cut set into an equilibrium load on the component. A special varient of this principle was used in [19].

We begin with the simple case where no vertices are added.

LEMMA 2.2 (Isostatic Substitution Principle) Given a geometric framework $\mathcal{F}=(G, \boldsymbol{p})=((V, E), \boldsymbol{p})$, with an isostatic subframework $\mathcal{F}^{\prime}=$ $\left(G^{\prime}, \boldsymbol{p}^{\prime}\right)$ on a set of vertices $V^{\prime} \subset V$, then replacing $\mathcal{F}^{\prime}=\left(G^{\prime},\left.\boldsymbol{p}\right|_{V^{\prime}}\right)$ with another isostatic subframework $\mathcal{F}^{\prime \prime}=\left(\left(V^{\prime}, E^{\prime \prime}\right),\left.\boldsymbol{p}\right|_{V^{\prime}}\right)$ on the same vertices $V^{\prime}$, gives a new framework $\mathcal{F}^{*}=\left(G^{*}, \boldsymbol{p}\right)=\left(\left(V, E^{*}\right), \boldsymbol{p}\right)$ which has the same space of resolvable loads $\mathcal{B}$, and an isomorphic space of self-stresses.

Proof. The core idea is that, working within a vector space of equilibrium loads, isostatic subframeworks are bases for the subspace of possible equilibrium loads on the vertices of the subframework. If $\mathcal{F}^{\prime}$ and $\mathcal{F}^{\prime \prime}$ use the same vertices $V^{\prime}$, then it is clear that we are replacing one basis for the space of equilibrium loads on $\left.\boldsymbol{p}\right|_{V^{\prime}}$ with another basis for this same subspace of equilibrium loads.

The larger vector space of resolved equilibrium loads on the entire framework $\mathcal{B}$ has the same dimension as $\mathcal{B}^{*}$. We have the same number of bars (basis are all the same size in a vector space) so we have with equivalent spaces of self-stress (dependencies of the bar loads).

We offer an extension of this, in which the substituted subframework may possess more vertices than the attaching set of vertices $V^{\prime}$. That is, the substituted framework has vertices $V^{\prime} \cup U$, where $U \cap V=\emptyset$. In particular, if $V^{\prime}$ is a set of block vertices, then an isostatic framework on these vertices may be substituted with another isostatic framework on the vertices $V^{\prime} \cup U$, while maintaining an isomorphic space of self-stresses.

COROLLARY 2.3 (General Isostatic Substitution Principle) Given a geometric framework $\mathcal{F}=(G, \boldsymbol{p})=((V, E), \boldsymbol{p})$, with an isostatic subframework $\mathcal{F}^{\prime}=\left(G^{\prime}, \boldsymbol{p}^{\prime}\right)$ on a set of vertices $V^{\prime} \subset V$, then replacing $\mathcal{F}^{\prime}=\left(G^{\prime},\left.\boldsymbol{p}\right|_{V^{\prime}}\right)$ with another isostatic subframework $\mathcal{F}^{\prime \prime}=\left(\left(V^{\prime} \cup U, E^{\prime \prime}\right),\left.\boldsymbol{p}\right|_{V^{\prime}} \cup \boldsymbol{q}\right)$ on the same vertices $V^{\prime}$, plus possible additional vertices $U$ in general position $\boldsymbol{q}$ relative to $\left.\boldsymbol{p}\right|_{V^{\prime}}$, gives a new framework $\mathcal{F}^{*}=\left(G^{*}, \boldsymbol{p}\right)=\left(\left(V \cup U, E^{*}\right), \boldsymbol{p} \cup \boldsymbol{q}\right)$ which has an isomorphic space of self-stresses.

Proof. If we have added vertices in $U$ which are not in $V^{\prime}$, then we can add these vertices to the original framework, at positions $\boldsymbol{q}$ by inserting general position 3 -valent vertices (non-coplanar) connected within $\mathcal{F}^{\prime}$ to get 
a modified framework and subframework with an isomorphic space of selfstresses [10]. Having done that to each of the pieces to be compared, we now have returned to the case of the previous lemma, and we have, overall, isomorphic spaces of unresolved equilibrium loads and of self-stresses. This will have a larger space of resolvable loads - it increased the dimension by $3|U|$.

This could be extended one more level - by having some vertices $W$ which are in $\mathcal{F}^{\prime}$ but not attached to the rest of $\mathcal{F}$, which are dropped, while new vertices $U$ are added. This level of generality is not needed here.

\subsection{Motions of Body and Hinge Structures}

It is traditional to analyze the rigidity of a framework with both the tools of static rigidity, as we have here, and tools of infinitesimal rigidity [8, 15, 14]. We will not present this kinematic theory for bar and joint frameworks here, as it is not needed.

An alternative approach to kinematics rigidity used in this paper, is the theory of infinitesimal motions for bodies and hinges. At a basic level, the motion of a framework or structure can be presented by velocity vectors assigned at all points of the structure in such a way as to not distort the geometric shape of the structure. That is, the velocity assignments must preserve the pairwise distances between points in the same rigid body of that structure. If the only such assignments correspond to rigid motions of the whole structure, then we say that the object is infinitesimally rigid.

In this section we focus on the structures of bodies and hinges we will use in the rest of this paper, using a projective algebra. A more detailed exposition, with examples and motivation, can be found in [3], and the Euclidean basis for this theory is described in [8].

A body and hinge structure $G^{*}(\boldsymbol{D})$ in $\mathbb{R}^{3}$ is a graph $G^{*}=(\mathcal{F}, \mathcal{D})$ together with a mapping $\boldsymbol{D}$ from $\mathcal{D}$ into the 2-extensors of $\mathbb{P}^{3}$. We think of the vertices, $F^{k} \in \mathcal{F}$ of $G^{*}$ as representing rigid bodies, and the edges $e \in \mathcal{D}$ represent hinges. If the vertices $F^{k} \in \mathcal{F}$ and $F^{m} \in \mathcal{F}$ are joined by an edge $e \in \mathcal{D}$, then we write $e=D^{k m}$. A body and hinge structure is connected if its underlying graph $G^{*}$ is connected.

A screw centre of motion for a body (a screw) is any vector $\mathbf{S} \in \mathbb{R}^{6}$. This 6 -vector represents a motion of $\mathbb{P}^{3}$, in that it encodes all the information for defining the velocities of any combination of rotations and translations. We can also interpret $\mathbf{S}$ as a weighted sum of 2-extensors in $\mathbb{P}^{3}$.

We note that the edge $D^{k m}$ of $G^{*}$ maps to a 2-extensor $\boldsymbol{D}^{k m}$ of $G^{*}(\boldsymbol{D})$, and we write $\boldsymbol{D}^{k m}=\mathbf{a} \vee \mathbf{b}$, for some $\mathbf{a}, \mathbf{b} \in \mathbb{P}^{3}$. Recall that $\mathbf{a} \vee \mathbf{b}=-(\mathbf{b} \vee \mathbf{a})$, 
and $\boldsymbol{D}^{m k}=\mathbf{b} \vee \mathbf{a}$, thus $\boldsymbol{D}^{k m}=-\boldsymbol{D}^{m k}$.

An infinitesimal motion of a body and hinge structure is an assignment of a screw centre $\boldsymbol{S}^{i}$ to each vertex $F^{k}$ of the graph $G^{*}$, such that for each edge $D^{k m} \in \boldsymbol{D}$,

$$
\boldsymbol{S}^{k}-\boldsymbol{S}^{m}=\omega^{k m} D^{k m}, \text { for some scalar } \omega^{k m} \in \mathbb{R} .
$$

We think of this equation as an assignment of a center for the rigid motion of each body of the structure, with the constraint that points along the hinge receive the same velocity from each of the adjacent centers.

Definition 2.4 A body and hinge structure is infinitesimally rigid if every infinitesimal motion is a rigid motion.

That is, it is infinitesimally rigid if every infinitesimal motion is trivial, with all vertices (bodies) receiving the same screw center assignment, $\boldsymbol{S}$. In this case the whole structure moves according to the motion encoded by $\boldsymbol{S}$.

There is a more condensed way of describing the motion of a given structure. A motion assignment is an assignment of scalars $\omega^{k m}$ to the hinges $\boldsymbol{D}^{k m}$ of the body and hinge structure such that:

1. $\omega^{k m}=\omega^{m k}$, and

2. $\sum \omega^{k m} \boldsymbol{D}^{k m}=\mathbf{0}$ for every closed cycle of panels and hinges in the structure.

The following relationship exists between the infinitesimal motions and the motion assignments of a body and hinge structure:

PROPOSITION 2.4 [3] For a connected body and hinge structure $G^{*}(\boldsymbol{D})$ with a designated body $F^{*} \in \mathcal{F}$, there is a one-to-one correspondence between the infinitesimal motions of the structure with $\boldsymbol{S}^{F^{*}}=\mathbf{0}$ and the motion assignments on the structure. A motion assignment represents a non-trivial motion if, and only if $\omega^{k m} \neq 0$ for some hinge $D^{k m}$.

Remark 1. Note that we can change the body we consider fixed in the above. That is, given an infinitesimal motion of the structure with $\boldsymbol{S}_{1}=\mathbf{0}$ and $\boldsymbol{S}_{m} \neq \mathbf{0}$, we can convert this to an equivalent motion with $\boldsymbol{S}^{m \prime}=\mathbf{0}$ by setting $\boldsymbol{S}_{k}{ }^{\prime}=\boldsymbol{S}_{k}-\boldsymbol{S}_{m}$. This new motion will generate the same scalars for the motion assignment, but we have a new frame of reference for the space. In the rest of this paper we will focus on the motion assignment as the record of the motion. 
Remark 2. We did not introduce the full vocabulary and notation for the projective analysis of infinitesimal motions of bar and joint frameworks in this section. In particular, we have not confirmed directly that the space of infinitesimal motions of a bar and joint framework is unchanged by isostatic substitution. Our static analysis, and associated vector spaces does include a space which is isomorphic to the space of non-trivial infinitesimal motions. Namely, if we take the orthogonal complement of the space of resolved loads $\mathcal{B}^{\perp}$ within the space of all equilibrium loads, we get a space $\mathcal{U}=\mathcal{B}^{\perp}$. This vector space has the same dimension as the space of non-trivial infinitesimal motions, as is verified in a more complete presentation [19, 14, 15].

We will not use any details of this proxy space below, but it is relevant to recognizing that the Isostatic Substitution Principle, Lemma 2.2, also shows that the substitution gives an isomorphism of the spaces of unresolved loads, or equivalently, an isomorphism of the spaces of non-trivial infinitesimal motions. The the proof of Corollary 2.3 also shows that such a substitution produces an an isomorphism of the spaces of unresolved loads, or equivalently, an isomorphism of the spaces of non-trivial infinitesimal motions.

Of course, our body-hinge structures do not depend on what is used to build the body, as long as each body in the structure is statically (infinitesimally) rigid.

\subsection{Notation and Connections on Block and Hole Polyhedra}

Informally, when we study a block and hole polyhedron as a bar and joint framework at $\boldsymbol{p}$ we want the block to be (statically) rigid. For each block $B_{\mathcal{P}}^{k}$ we introduce some added subframework $I^{k}=\left(V^{k}, U^{k}, E^{k}\right)$ where $V^{k}$ is the set of vertices of the block face, $U^{k}$ are some added new vertices (possibly empty), and $E^{k}$ are some added edges on $V^{k} \cup U^{k}$ such that, with the edges of the polygon added, $I^{k}$ becomes an isostatic bar and joint framework realized at $\left.\boldsymbol{p}\right|_{V^{k}}$ with generic positions for $U^{k}$.

We note that if all vertices of a block face are collinear, then there is no possible $I^{k}$ making an isostatic framework, since this collinear polygon is already dependent. If the vertices of a block face boundary are coplanar, then we will need to add some vertex in $U^{k}$ off the plane in order to achieve an isostatic subframework. If the vertices of the block face boundary polygon are not coplanar, then we can create $I^{k}$ with only added edges and no added vertices.

The key properties of the block and hole frameworks do not depend on which isostatic subframework is inserted for each block, provided that the boundary polygon of the original face is used as part of the framework. This 
is captured by the Isostatic Substitution Principle given in Lemma 2.2.

With this in mind, in the remainder of this paper we will be non-specific about which isostatic subframework is used in place of a block, with the exception that we do assume that the original polygon of the face is present among the edges of the isostatic framework.

Definition 2.5 The static framework graph, $G_{S}(\mathcal{P})$, is the graph of a block and hole polyhedral framework with the added frameworks $I^{k}$ for each block. Since we do not pay attention to the isostatic subframeworks on the blocks, we consider $G_{S}(\mathcal{P})$ to be a representative framework among an equivalence class of graphs (with various isostatic blocks inserted into the block faces).

This graph $G_{S}(\mathcal{P})$ will be used to track the stresses of such frameworks on $\mathcal{P}$. The graphs $G_{S}(\mathcal{P})$ and $G_{S}(\overline{\mathcal{P}})$ exist for every block and hole polyhedron (see Figure 4. (c) and (d)). At a configuration $\boldsymbol{p}$ they form bar and joint frameworks with well defined spaces of self-stresses, which we denote $\mathcal{S}\left(G_{S}(\mathcal{P}, \boldsymbol{p})\right), \mathcal{S}\left(G_{S}(\overline{\mathcal{P}}, \boldsymbol{p})\right)$. The space of residual unresolved equilibrium loads for these frameworks (our proxy space for the bar and joint infinitesimal motions), is denoted by $\mathcal{M}\left(G_{S}(\mathcal{P}, \boldsymbol{p})\right), \mathcal{M}\left(G_{S}(\overline{\mathcal{P}}, \boldsymbol{p})\right)$.

At the heart of this paper, we explore the connections (essentially an isomorphism for appropriate polyhedra) between the space $\mathcal{M}\left(G_{S}(\mathcal{P}, \boldsymbol{p})\right)$ for the original polyhedron and the stress space $\mathcal{S}\left(G_{S}(\overline{\mathcal{P}}, \boldsymbol{p})\right)$ for the swapped polyhedron.

As an intermediary in the proofs, we will use an induced body and hinge structure on $(\mathcal{P}, \boldsymbol{p})$ in place of $\mathcal{M}\left(G_{S}(\mathcal{P}, \boldsymbol{p})\right)$ to track these connections. This body and hinge structure is composed of the rigid bodies (surface faces and bodies, but not holes), and edges between rigid faces of the underlying spherical block and hole polyhedron $\mathcal{P}$ to form the body and hinge polyhedron $G^{M}(\mathcal{P})$ (Figure 4. (e) and (f)). For a particular configuration $\boldsymbol{p}$, we denote the vector space of motion assignments on this structure by $\mathcal{M}\left(G^{M}(\mathcal{P}, \boldsymbol{p})\right)$. As we will see, for block and hole polyhedra $\mathcal{P}$ satisfying certain conditions, the spaces $\mathcal{M}\left(G^{M}(\mathcal{P}, \boldsymbol{p})\right)$ and $\mathcal{M}\left(G_{S}(\mathcal{P}, \boldsymbol{p})\right)$ are isomorphic. We first address the situations in which these spaces are not isomorphic.

The structure $G^{M}(\mathcal{P}, \boldsymbol{p})$ may not form a sufficiently connected graph for the body and hinge structure to have only the motions of the underlying bar and joint framework, which make up the vector space $\mathcal{M}\left(G_{S}(\mathcal{P}, \boldsymbol{p})\right)$. Figure 5 (a) and (b) illustrate this with a polyhedron $\mathcal{P}$ for which the body-hinge structure $G^{M}(\mathcal{P})$ is disconnected. Figure 5 (c) and (d) depict a related example where the connectivity is sufficient to capture the motions of the underlying bar and joint framework. This problem of connectivity arises 


\begin{tabular}{|l|l|}
\hline 1. 5-gon block, & 2.gon block, \\
4-gon hole
\end{tabular}

Figure 4: Examples of block and hole polyhedra, and their associated graphs $G_{S}(\mathcal{P})$ and $G^{M}(\mathcal{P})$. 
only when $\mathcal{P}$ has a vertex with two or more holes at the vertex, as in figure 5 (a) and (b). The graph of rigid faces and shared edges $G^{M}(\mathcal{P})$, no longer capture the nature of the motions of the rigid faces at this vertex. As such, the creation of the graph $G^{M}(\mathcal{P})$ may introduce extra motions not in the underlying framework. This observation motivates the following definition:

Definition 2.6 A block and hole polyhedra $\mathcal{P}$ is separated if there is at most one hole at any given vertex. That is, there are no contact vertices where several holes meet.

PROPOSITION 2.5 If a polyhedron $\mathcal{P}$ is separated, then the spaces $\mathcal{M}\left(G_{S}(\mathcal{P}, p)\right)$ and $\mathcal{M}\left(G^{M}(\mathcal{P}, \boldsymbol{p})\right)$ are isomorphic.

Proof. We will only sketch the outline of this connection, as we have not developed the full background of infinitesimal motions for bar and joint frameworks, or even the velocities at points induced by the centers of motion. The result is implicit in $[3]$.

The general observation is that every motion of the bar and joint framework will induce velocities which correspond to the centers of motion for the rigid faces. There is an injection from $\mathcal{M}\left(G_{S}(\mathcal{P}, \boldsymbol{p})\right)$ into $\mathcal{M}\left(G^{M}(\mathcal{P}, \boldsymbol{p})\right)$.

If the polyhedron is separated, then each motion in $\mathcal{M}\left(G^{M}(\mathcal{P}, \boldsymbol{p})\right)$ induces a set of unique velocities for each of the vertices in $\boldsymbol{p}$, and therefore a unique corresponding motion in $\mathcal{M}\left(G_{S}(\mathcal{P}, \boldsymbol{p})\right)$. This completes the isomorphism.

Figure 4 shows the two graphs $G^{M}$ and $G_{S}$ for both a block and hole polyhedron $\mathcal{P}$ and its swapped form, $\overline{\mathcal{P}}$. It is the connections among these four structures which will be at the heart of the analysis in Section 3 .

We note that the swapping induces a map of the related graphs: $G^{M}(\overline{\overline{\mathcal{P}}})=G^{M}(\mathcal{P})$. Moreover $G_{S}(\overline{\overline{\mathcal{P}}})$ is equivalent to $G_{S}(\mathcal{P})$. That is, they are the same graphs up to an interchange of which isostatic subframeworks are inserted into the blocks of $\mathcal{P}$.

Our analysis will build up the complete set of connections for separated block and hole polyhedra (Section 3). In Section 4 we will describe an operation which takes a general (non-separated) block and hole polyhedron to an extended (gusseted) block and hole polyhedron with equivalent spaces of stresses and motions as bar and joint frameworks. 


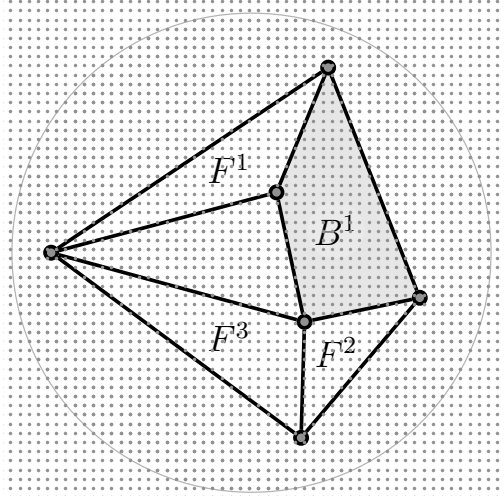

(a) $\mathcal{P}$

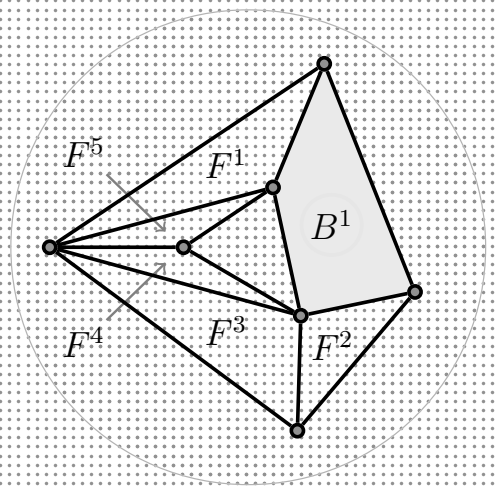

(c) $\mathcal{P}^{\prime}$

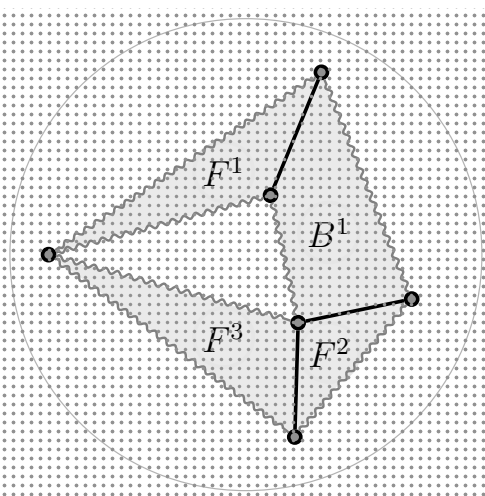

(b) $G^{M}(\mathcal{P})$

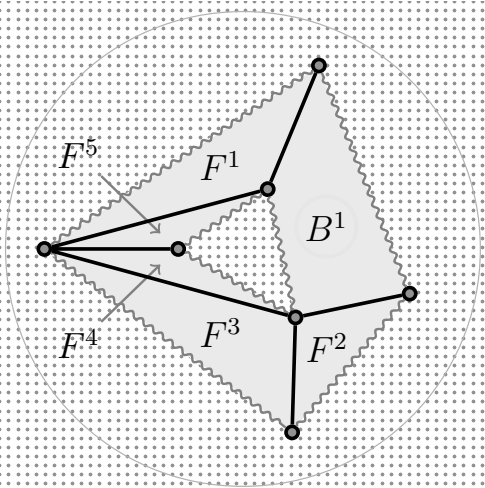

(d) $G^{M}(\mathcal{P})$

Figure 5: If we have a vertex in contact with two holes as in (a), the graph $G^{M}(\mathcal{P})$ shown in (b) does not track the motion of $\mathcal{P}$. The graph $\mathcal{P}^{\prime}$ shown in (c) has an isomorphic space of stresses, and now $G^{M}\left(\mathcal{P}^{\prime}\right)$ shown in (d) does capture the motions. 


\subsubsection{Example}

A tower is a block and hole polyhedra with one $s$-gon block and one $t$-gon hole. In this example, we consider several of the towers described in [5], and investigate the impact of swapping on the spaces of self-stresses and motion to get an idea of what to anticipate in the later proofs. We will assume there are $\min (s, t)$ edge disjoint paths between this block and hole, in order to give simple predictions for the anticipated spaces of stresses and first-order motions (see [5]). (See Figure 4)

1. If $s>t$, then we have implicitly started with a triangulated sphere (with $|E|=3|V|-6$ ), removed $t-3$ edges from the hole and added $s-3$ edges to make the block polygon (already triangulated) into an isostatic subgraph. The net effect is that $\left|E^{\prime}\right|=3|V|-6+[s-t]>$ $3|V|-6$. This will have more bars than needed for first-order rigidity, so we will have a space of self-stresses of dimension at least $s-t$. In generic realizations, we will have only the trivial motion assignment.

2. If $s<t$, then we have started with a triangulated sphere, removed $t-3$ edges from the hole and added $s-3$ edges to make the block polygon (triangulated) into an isostatic subgraph. The net effect is that $\left|E^{\prime}\right|=3|V|-6-[t-s]<3|V|-6$. This will have fewer bars than needed to resolve all equilibrium loads, so we will have a space of internal motions of dimension at least $t-s$. In generic realizations, the bars will be independent, and we will have only the zero dimensional space of trivial self-stresses.

This structure is really a swapped polyhedron from case (i), where $s=t$ and $t=s$. Translating, we see that the dimension of the space of internal motions after swapping is the same dimension as the space of self-stresses before swapping, and that the space of self-stresses after swapping is the same dimension as the space of internal motions before swapping.

3. If $s=t$, then making the hole removes $s-3$ edges from a triangulated sphere, and adds $s-3$ edges to make the block polygon (triangulated) into an isostatic subgraph. We return to a collection of edges with the desired $|E|=3|V|-6$. In generic position, this will be isostatic with only the trivial self-stress, and only the trivial motion assignment. The swapped polyhedron will have the same overall counts, and the dimensions of stresses and motion assignments will match after swapping. 
From the example, we can anticipate that swapping will take internal motions of the original block and hole polyhedra to self-stresses of the swapped structure, both generically, and in special position geometrically. This is a good set of elementary examples to keep in mind in the next few sections.

\section{Motions and Stresses in Separated Spherical Structures}

In this section, we prove our main result for the case of separated block and hole polyhedra in which there is at most one block and at most one hole at any given vertex. This is sufficient to guarantee that the graphs $G^{M}(\mathcal{P})$, as well as $G^{M}(\overline{\mathcal{P}})$, are connected and that these graphs provide full information about the rigidity of the underlying block and hole polyhedron. While there are block and hole polyhedra with contact vertices, it is helpful to cluster all of the extensions to more general block and whole polyhedra into the next section, where a single added technique captures all these extensions.

As our results are geometric in nature, we will be working with specific realizations of block and hole polyhedra as bar and joint frameworks (for $G_{S}(\mathcal{P})$ ) or as hinged panel structures (for $G^{M}(\mathcal{P})$ ). When a projective embedding $\boldsymbol{p}$ of the graph is given, we write $G^{M}(\mathcal{P}, \boldsymbol{p})$ or $G_{S}(\mathcal{P}, \boldsymbol{p})$ to denote the particular configuration in $\mathbb{P}^{3}$ viewed as a body and hinge structure or a bar and joint framework, respectively. Note that the key rigidity properties, namely static rigidity, infinitesimal rigidity, and independence, are projectively invariant; (see[3], $\S 4.2$ ).

In a specific geometric realization, we will restrict the choices of the block subgraph in $G_{S}(\mathcal{P}, \boldsymbol{p})$ to those which are isostatic in that realization. For this reason, in order to have some isostatic subgraphs available, we will assume that the configurations used do not have all vertices of a block face (and because of swapping, of a hole) collinear. We call such configurations block and hole general position.

We develop the proof of the main theorem through two propositions.

PROPOSITION 3.1 Given a separated block and hole polyhedron $\mathcal{P}$, there is injective map from the stresses of the bar and joint framework $G_{S}(\overline{\mathcal{P}}, \boldsymbol{p})$ to the motion assignments of the hinged panel structure of the swapped polyhedron at the same configuration, $G^{M}(\mathcal{P}, \boldsymbol{p})$.

PROPOSITION 3.2 Given a separated block and hole polyhedron $\mathcal{P}$, there is an injective map from the motion assignments of the hinged panel 
structure $G^{M}(\mathcal{P}, \boldsymbol{p})$ to the stresses of the swapped block and hole structure as a bar and joint framework at the same configuration, $G_{S}(\overline{\mathcal{P}}, \boldsymbol{p})$.

Together, these propositions and their proofs will yield the main theorem for separated polyhedra:

THEOREM 3.3 Given a separated block and hole polyhedron, $\mathcal{P}$, there is an isomorphism between the space of motion assignments of the swapped block and hole structure $G^{M}(\overline{\mathcal{P}}, \boldsymbol{p})$ as a hinged panel polyhedron and the space of stresses of the bar and joint framework at the same configuration, $G_{S}(\mathcal{P}, \boldsymbol{p})$. That is, $\mathcal{S}\left(G_{S}(\mathcal{P}, \boldsymbol{p})\right) \simeq \mathcal{M}\left(G^{M}(\overline{\mathcal{P}}, \boldsymbol{p})\right)$.

\subsection{From Stresses to Motions in Separated Block and Hole Polyhedra}

Proof of Proposition 3.1 for separated block and hole polyhedra.

Let $\overline{\mathcal{P}}$ be a separated block and hole polyhedron with blocks $\mathcal{B}=$ $\left\{B_{\overline{\mathcal{P}}}^{1}, \ldots B_{\overline{\mathcal{P}}}^{\ell}\right\}$ and holes $\mathcal{H}=\left\{H_{\overline{\mathcal{P}}}^{1}, \ldots H_{\overline{\mathcal{P}}}^{n}\right\}$, and consider the graph $G_{S}(\overline{\mathcal{P}}, \boldsymbol{p})$, where $\boldsymbol{p}$ is some embedding of the graph into $\mathbb{P}^{3}$.

Suppose we have a non-trivial stress $\Lambda$ on $G_{S}(\overline{\mathcal{P}}, \boldsymbol{p})$, given by scalars $\left(\ldots, \lambda_{i j}, \ldots\right)$. From the stress, we define a motion assignment on the swapped framework $G^{M}(\mathcal{P}, \boldsymbol{p})$ by setting $\omega^{k m}=\lambda_{i j}$ if $\left\langle v_{i}, v_{j} ; F^{k}, F^{m}>\right.$ is an edge patch. This map ignores the scalars within, or on the boundary of any block of $G_{S}(\overline{\mathcal{P}}, \boldsymbol{p})$ as a block $B_{\overline{\mathcal{P}}}^{k}$ in $G_{S}(\overline{\mathcal{P}}, \boldsymbol{p})$ becomes the hole $H_{\mathcal{P}}^{k}$ in the swapped polyhedron and the edges are not in $G^{M}(\mathcal{P}, \boldsymbol{p})$

To show that the new set of scalars $\left(\ldots, \omega^{m k}, \ldots\right)$ is indeed a valid motion assignment to the hinges $\left(\ldots, \boldsymbol{D}^{m k}, \ldots\right)$, we first prove that there is exactly one scalar for each edge; that is, $\omega^{m k}=\omega^{k m}$. To see this, note that the scalar assignment $\lambda_{i j}$ to the bar $\boldsymbol{p}_{i} \boldsymbol{p}_{j}$ must be balanced by the scalar $\lambda_{j i}$ on the bar $\boldsymbol{p}_{j} \boldsymbol{p}_{i}$. That is, $\lambda_{i j}\left(\boldsymbol{p}_{i} \boldsymbol{p}_{j}\right)=-\lambda_{j i}\left(\boldsymbol{p}_{j} \boldsymbol{p}_{i}\right)$. Since $\boldsymbol{p}_{i} \boldsymbol{p}_{j}=-\left(\boldsymbol{p}_{j} \boldsymbol{p}_{i}\right)$, $\lambda_{i j}\left(\boldsymbol{p}_{i} \boldsymbol{p}_{j}\right)=\lambda_{j i}\left(\boldsymbol{p}_{i} \boldsymbol{p}_{j}\right)$, and it follows that $\omega^{m k}=\lambda_{i j}=\lambda_{j i}=\omega^{k m}$.

Next, we show that given any simple closed cycle $\mathcal{Z}=$ $F^{0} \boldsymbol{D}^{01} F^{1} \boldsymbol{D}^{12} F^{2} \cdots F^{k} \boldsymbol{D}^{k 0}$ of panels and hinges in the swapped polyhedral structure $G^{M}(\mathcal{P}, \boldsymbol{p})$, we have

$$
\sum_{\boldsymbol{D}^{k m} \in \mathcal{Z}} \omega^{k m} \boldsymbol{D}^{k m}=\mathbf{0}
$$

where the sum is over each hinge in the cycle. Note that such a cycle necessarily does not cross any holes as faces. 
Because $G^{M}(\mathcal{P})$ is a part of the spherical polyhedron, any simple closed cycle of panels and hinges (possibly including blocks) will disconnect the polyhedron, and the graph $G_{S}(\overline{\mathcal{P}})$. Since $\omega^{k m}=\lambda_{i j}, \boldsymbol{D}^{k m}=\boldsymbol{p}_{i} \boldsymbol{p}_{j}$, and the collection of edges $\left\{v_{i}, v_{j}\right\}$ is a cut set, Theorem 2.1 yields:

$$
\begin{aligned}
\sum_{\boldsymbol{D}^{k m} \in \mathcal{Z}} \omega^{k m} \boldsymbol{D}^{k m} & =\sum_{i=1}^{n}\left(\sum_{\substack{\left\{j \mid \boldsymbol{D}^{k m} \in \mathcal{Z}, \boldsymbol{D}^{k m}=\boldsymbol{q}_{i} \boldsymbol{p}_{j}\right\}}} \lambda_{i j} \boldsymbol{p}_{i} \boldsymbol{p}_{j}\right) \\
& =0 .
\end{aligned}
$$

Since both conditions of a motion assignment are satisfied, a stress in a bar and joint framework induces a motion assignment in the swapped panel polyhedron.

To see that this injective, we need to check that distinct self-stresses induce distinct motion assignments. Suppose there are two distinct selfstreses $\Lambda$ and $\Lambda^{\prime}$ with $\lambda_{i j} \neq \lambda_{i j}^{\prime}$ for some block edge, and all non-block edges have trivial (zero) scalars. Since the polyhedron is separated, no two blocks share a vertex and hence the self-stress must be contained on a single block. But each block has an isostatic framework (independent) and therefore contains no self-stress, which provides the contradiction. We conclude that the self-stresses $\Lambda$ and $\Lambda^{\prime}$ must differ on some edges which induce distinct scalars for the motion assignment.

The correspondence is actually a linear transformation of the two vector spaces, as the sum of two stresses goes to the sum of the corresponding motion assignments and a scalar multiple of a stress goes to the scalar multiple of the motion assignment.

COROLLARY 3.4 Given a separated block and hole polyhedron $\mathcal{P}$, there is injective linear transformation from the space of stresses of the bar and joint framework $G_{S}(\mathcal{P}, \boldsymbol{p})$ to the space of motion assignments of the hinged panel structure of the swapped polyhedron at the same configuration, $G^{M}(\overline{\mathcal{P}}, \boldsymbol{p})$.

\subsection{From Motions to Stresses in Separated Block and Hole Polyhedra}

We now give the proof of the converse result. 
Proof of Proposition 3.2 for separated block and hole polyhedra

Let $\mathcal{P}$ be a separated block and hole polyhedron with blocks $\mathcal{B}=$ $\left\{B_{\mathcal{P}}^{1}, \ldots B_{\mathcal{P}}^{\ell}\right\}$ and holes $\mathcal{H}=\left\{H_{\mathcal{P}}^{1}, \ldots H_{\mathcal{P}}^{n}\right\}$, and consider the graph $G^{M}(\mathcal{P}, \boldsymbol{p})$, where $\boldsymbol{p}$ is some embedding of the graph into $\mathbb{P}^{3}$.

Suppose we have some non-trivial motion assignment $\left(\ldots \omega^{k m} \ldots\right)$ on the graph $G^{M}(\mathcal{P}, \boldsymbol{p})$. Consider the swapped block and hole polyhedral framework $\overline{\mathcal{P}}$, and set $\lambda_{i j}=\omega^{k m}$ whenever $\left\langle v_{i}, v_{j} ; F^{k}, F^{m}>\right.$ is an edge patch. The result is an assignment of scalars to some of the bars of $G_{S}(\overline{\mathcal{P}}, \boldsymbol{p})$. We show that this can be extended to a stress on all the bars of $G_{S}(\overline{\mathcal{P}}, \boldsymbol{p})$. In particular, we show that we can assign scalars to the remaining bars such that for each vertex of $\overline{\mathcal{P}}$, Equation $(3)$ is satisfied.

Since $\mathcal{P}$ is separated, $\overline{\mathcal{P}}$ is also separated. Therefore any vertex, $v_{i}$ of $\overline{\mathcal{P}}$ is one of the following two distinct types: it is either adjacent to a single block (called a block vertex), or $a_{i}$ is adjacent to no block of $\overline{\mathcal{P}}$.

In the latter case, there is a simple closed cycle of panels and oriented hinges in $G^{M}(\mathcal{P}), \mathcal{Z}=F^{0} \boldsymbol{D}^{01} F^{1} \boldsymbol{D}^{12} F^{2} \cdots F^{k} \boldsymbol{D}^{k 0}$ around $v_{i}$. Since $\lambda_{k m}=$ $\omega^{i j}, \boldsymbol{D}^{k m}=\boldsymbol{p}_{i} \boldsymbol{p}_{j}$, and $\sum \omega^{k m} \boldsymbol{D}^{k m}=\mathbf{0}$ for the cycle around $v_{i}$,

$$
\sum_{i=1}^{n}\left(\sum_{\substack{\left\{j \mid \boldsymbol{D}^{k m} \in \mathcal{Z}, \boldsymbol{D}^{k m}=\boldsymbol{q}_{i} \boldsymbol{p}_{j}\right\}}} \lambda_{i j} \boldsymbol{p}_{i} \boldsymbol{p}_{j}\right)=\mathbf{0}
$$

holds in $\overline{\mathcal{P}}$. Hence, Equation (3) holds for all non-block vertices.

It remains to extend the scalars $\left(\ldots, \lambda_{i j}, \ldots\right)$ to the edges of the blocks in $\overline{\mathcal{P}}$, and to show that the block vertices satisfy Equation (3). Because $\mathcal{P}$ is a separated block and hole polyhedron, there is a simple closed cycle of panels and hinges, $\mathcal{Z}=\left(F^{0} \boldsymbol{D}^{01} F^{1} \boldsymbol{D}^{12} F^{2} \cdots F^{k} \boldsymbol{D}^{k 0}\right)$, around the boundary of every hole in $G^{M}(\mathcal{P}, \boldsymbol{p})$ (i.e. every block of $\overline{\mathcal{P}}$ ). The cycle satisfies the cycle condition $\sum_{\boldsymbol{D}^{k m} \in \mathcal{Z}} \omega^{k m} \boldsymbol{D}^{k m}=\mathbf{0}$. Consider the hole $H_{\mathcal{P}}^{i}$ with vertices $J=\left\{b_{1}, \ldots, b_{n}\right\}$ and let $\mathcal{Z}$ be the set of oriented hinges into the hole vertices $b_{i}$ from adjacent non-hole vertices $a_{j}$. Under swapping, this property of the holes of $\mathcal{P}$ translates to a property about the blocks in $\overline{\mathcal{P}}$. Indeed, $B \frac{i}{\mathcal{P}}$ satisfies

$$
\sum_{i=1}^{n}\left(\sum_{\substack{\left\{j \mid \boldsymbol{D}^{k m} \in \mathcal{Z}, \boldsymbol{D}^{k m}=\boldsymbol{q}_{i} \boldsymbol{p}_{j}\right\}}} \lambda_{i j} \boldsymbol{q}_{i} \boldsymbol{p}_{j}\right)=\mathbf{0}
$$


Equation (4) implies that the forces on the block vertices form an equilibrium load. Since each block is built as an isostatic subframework, the block is statically rigid, and therefore its edges will resolve any equilibrium load on the block. Furthermore, because the block is isostatic and therefore independent, there is in fact a unique assignment of scalars to the block edges that resolves this load. These scalars are the missing $\lambda_{i j}$ 's. We now have exactly one scalar for every edge of $G_{S}(\overline{\mathcal{P}}, \boldsymbol{p})$. The resolution of the stress in the block guarantees that the equilibrium condition (Equation (3)) is satisfied for each of the block vertices.

Note that the zero motion assignment goes to the zero self-stress, as expected. If we have two different motion assignments with $\omega^{k, m} \neq \theta^{k, m}$, clearly the induced self-stresses are zero, so the map is injective.

Again, this map is a linear transformation.

COROLLARY 3.5 Given a separated block and hole polyhedron $\mathcal{P}$, there is an injective map from the space of motion assignments of the hinged panel structure $G^{M}(\mathcal{P}, \boldsymbol{p})$ to the space of stresses of the swapped block and hole structure as a bar and joint framework at the same configuration, $G_{S}(\overline{\mathcal{P}}, \boldsymbol{p})$.

\subsection{Main result and corollaries}

Section 3.1 completed the proof of Proposition 3.1 for separated block and hole polyhedra and Section 3.2 completed the proof of Proposition 3.2. The corollaries extended these results to demonstrate isomorphism of the corresponding vector spaces.

There are some geometric corollaries, as well as some generic corollaries of these two propositions and Theorem 3.3 .

COROLLARY 3.6 The vector space of stresses on a separated block and hole polyhedral framework $G_{S}(\mathcal{P}, \boldsymbol{p})$ is isomorphic to the vector space of motion assignments of the swapped block and hole polyhedron $G^{M}(\overline{\mathcal{P}}, \boldsymbol{p})$.

\section{General Block and Hole Polyhedra}

Following the proofs for separated block and hole polyhedra, in this section we extend the results to more general block and hole polyhedra that have adjacent holes or adjacent blocks. We assume that the configurations used in these geometric results do not have three adjacent vertices in any block or hole collinear, that is, that the configurations are in block and hole general 
position. This is not needed for separated polyhedra, where no vertex lies on more than one block or hole.

\subsection{Gussets to create separated block and hole polyhedra}

In this subsection, we will introduce gussets; gussets add some edges and triangles to separate the original block and hole polyhedron, while extending the stresses and motions of the original polyhedron to isomorphic spaces on the extended polyhedron. In addition, gussetting and swapping are commutative operations (that is, first gussetting then swapping will yield identical results to swapping then gussetting). The gussetting procedure will allow us to extend Theorem 3.3 to general spherical block and hole polyhedra. Figure 5 provides an intuition for the gusseting procedure.

Throughout this section, we assume $\boldsymbol{p}$ is in block and hole general position for a given polyhedron $\mathcal{P}$. Thus, in the following constructions a set of three vertices $\left\{u_{-1}, u, u_{+1}\right\}$, where $\left\{u_{-1}, u\right\}$ and $\left\{u, u_{+1}\right\}$ are adjacent vertices along the same block or hole, are not collinear. We refer to the embedded graph of the polyhedron $G(\mathcal{P}, \boldsymbol{p})=G(\boldsymbol{p})$ as a block and hole polyhedron, or simply as a polyhedron.

Definition 4.1 Given a non-separated vertex $u$ on a hole $H$ of a block and hole polyhedron $G(\boldsymbol{p})$ such that the two pairs $u_{-1}, u$, and $u, u_{+1}$ are adjacent vertices along $H$, a hole-gusseted polyhedron, $G^{\prime}\left(\boldsymbol{p}^{\prime}\right)$, is the extension of $G$ by the addition of a new 3 -valent vertex $v_{u}$ which is attached by three edges, $\left(v_{u}, u_{-1}\right),\left(v_{u}, u\right)$, and $\left(v_{u}, u_{+1}\right)$, creating the new hole $H^{\prime}$. Geometrically, we ensure that the new vertex is assigned a position $p_{v_{u}}$ in $\boldsymbol{p}^{\prime}$ which is not coplanar with the three attaching vertices. (See Figure 6.)

This construction is essentially what is traditionally called 3 -valent addition for bar and joint frameworks. However, what is different here is that we also extend the underlying polyhedron and regard $\left\{u_{-1}, u, v_{u}\right\}$ and $\left\{v_{u}, u, u_{+1}\right\}$ as two new triangular surface faces of the polyhedral structure. Notice that the addition of a gusset does not change the size of the hole, it just shifts the hole away from $u$ to $v_{u}$, creating a new hole $H^{\prime}$.

PROPOSITION 4.1 Provided that $\boldsymbol{p}_{v_{u}}$ is not coplanar with $\boldsymbol{p}_{u_{-1}}, \boldsymbol{p}_{u}$, and $\boldsymbol{p}_{u_{+1}}$ as we create $\boldsymbol{p}^{\prime}$, there is a linear isomorpism between the space of stresses of $G(\boldsymbol{p})$ and the space of stresses of $G^{\prime}\left(\boldsymbol{p}^{\prime}\right)$ with the added hole-gusset, and between the motion assignments of $G(\boldsymbol{p})$ and the motion assignments of $G^{\prime}\left(\boldsymbol{p}^{\prime}\right)$ with the added hole-gusset. 


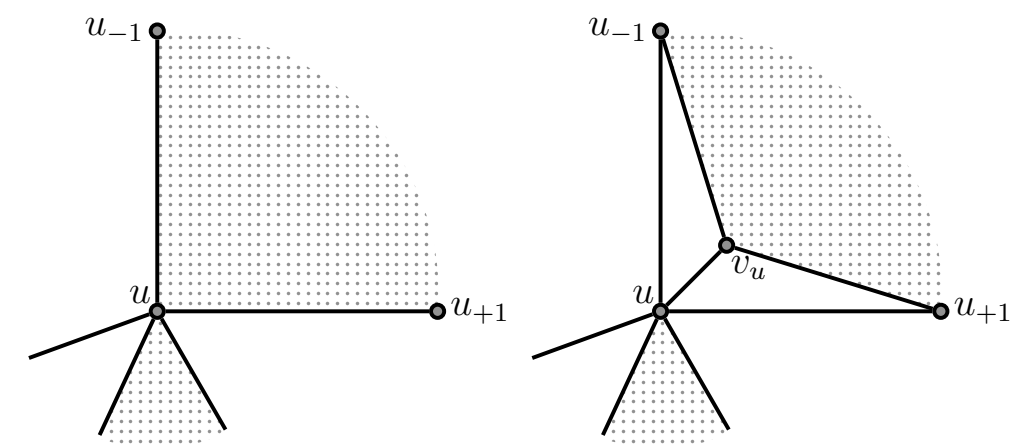

Figure 6: A non-separated hole vertex (left) becomes a non-contact vertex with the addition of a gusset (right).

Proof. The new 3-valent vertex (not coplanar with the vertices of attachment) cannot support a non-zero stress on its edges, so the space of stresses is unchanged [10]. This shows the isomorphism of the stresses.

We have added a 3 -valent vertex, which is independent. Therefore, by basic operations on the underlying framework, the space of first-order motions is also unchanged [10]. Given any first-order motion of $G(\boldsymbol{p})$ there is a unique velocity assigned to $p_{v_{u, H}}$ which extends the first-order motion. This in turn gives an extension of the motion assignment, leaving all previous scalars unchanged and generating new motion assignment scalars for the new edges between faces of the polyhedron: $\left\{u_{-1}, u\right\},\left\{u, v_{u}\right\}$ and $\left\{u, u_{+1}\right\}$. Again the uniqueness gives us the one-to-one correspondence of the spaces of motion assignments.

Next, we assume that $u$ is a non-separated vertex on a block, $B$. For defining a block gusset we have three goals:

1. we should make a new block which is no longer directly in contact with the vertex $u$, by inserting some triangular faces and a new vertex $v_{u}$;

2. this addition of a new vertex should be equivalent, after swapping, to adding a gusset to the hole in the swapped structure, and

3. the isostatic graph used for the block should be unchanged, with the new vertex $v_{u}$ replacing the vertex $u$.

The second objective gives us a clear picture of what we should do. 

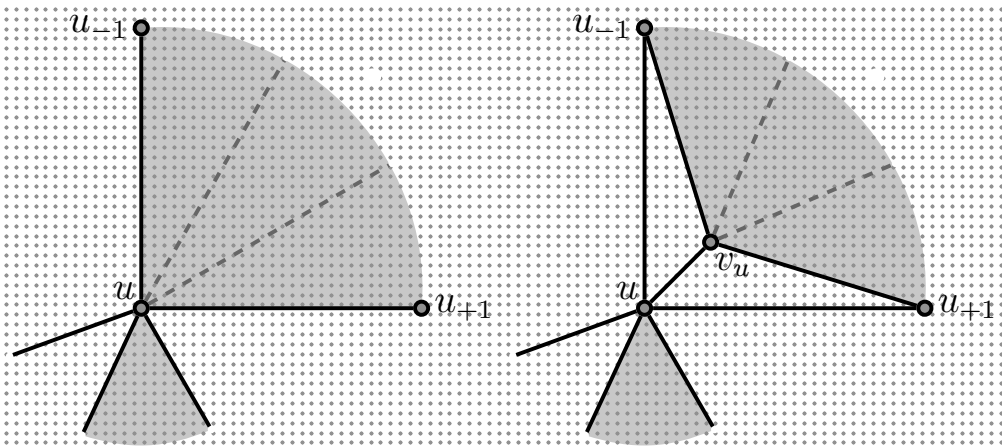

Figure 7: A gusset added at a non-separated block vertex.

Definition 4.2 Given a non-separated vertex $u$ on a block $B$ of a block and hole polyhedron $G(\boldsymbol{p})$, the block-gusseted polyhedron $G^{\prime}\left(\boldsymbol{p}^{\prime}\right)$ is the extension of $G$ by the vertex split of $u$ to $\left\{u, v_{u}\right\}$ along the edges $\left\{u_{-1}, u\right\}$ and $\left\{u, u_{+1}\right\}$, with all the block edges adjacent to $u$ in $G(\boldsymbol{p})$ becoming adjacent to $v_{u}$ in $G^{\prime}\left(\boldsymbol{p}^{\prime}\right)$, instead. (See Figure 7.)

Geometrically, we ensure that the new vertex is assigned a position $p_{v_{u}}$ in $\boldsymbol{p}^{\prime}$ which keeps the isostatic block framework of $B^{\prime}$ isostatic, and is also not coplanar with the three vertices of the split. This is always possible, by general considerations of avoiding the thin set of special positions for any particular graph vertices; cf [13]. We call such a position general for $(u, B)$.

PROPOSITION 4.2 There is an isomorphism between the space of stresses of $G(\boldsymbol{p})$ and the space of stresses of $G^{\prime}\left(\boldsymbol{p}^{\prime}\right)$ with the the added blockgusset, and between the space of motion assignments of $G(\boldsymbol{p})$ and the space motion assignments of $G^{\prime}\left(\boldsymbol{p}^{\prime}\right)$ with the the added block-gusset.

Proof. We have effectively replaced a general isostatic block framework on $B$ with a specific type of isostatic block framework in which we have added one new vertex $v_{u, B}$ and have ensured that $u$ is 3 -valent in the subframework, attached only to $u_{-1}, u_{+1}$ and $v_{u}$, and not coplanar with these points. By the Isostatic Substitution Principle, there is no change in the space of stresses or motions of the structure.

Now, given any vertex in contact with more than one hole or block, we insert gussets at this vertex into all but one of the holes and all but one of the blocks at that vertex. Repeat this for each such vertex, in sequence, 


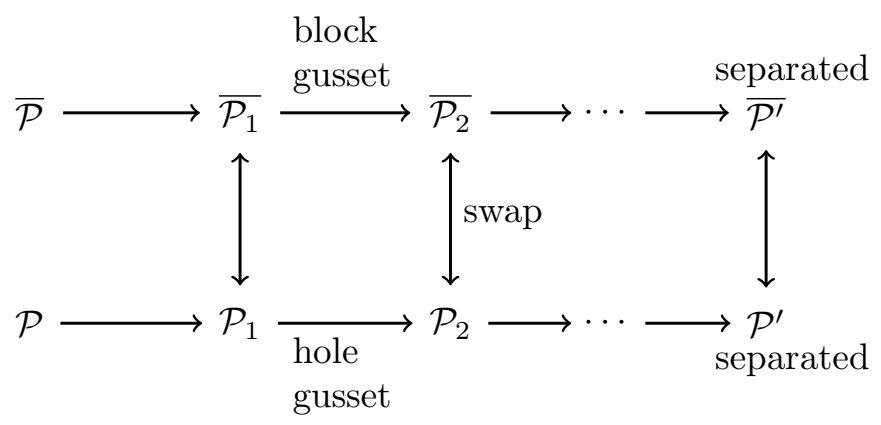

Figure 8: The gusseting and swapping processes.

and the resulting structure will be a separated block and hole polyhedron, as shown in Figure 8.

LEMMA 4.3 Given a block and hole polyhedron $G(\mathcal{P}, \boldsymbol{p})$, adding a holegusset at the vertex $u$ on a hole $H$ and swapping, creates the same structure as swapping to $G(\overline{\mathcal{P}}, \boldsymbol{p})$, and adding a block-gusset at the vertex $u$ on the block $\bar{H}$, provided we assign the same position to the added vertex, in creating $\boldsymbol{p}^{\prime}$.

Given a correspondence of the motion assignment on $G^{\prime}\left(\mathcal{P}^{\prime}, \boldsymbol{p}^{\prime}\right)$ with the stresses of $G^{\prime}\left(\overline{\mathcal{P}^{\prime}}, \boldsymbol{p}^{\prime}\right)$, there is an induced correspondence of the motion assignments on $G(\mathcal{P}, \boldsymbol{p})$ with the stresses of $G(\overline{\mathcal{P}}, \boldsymbol{p})$.

Notice that we will need to pick the new point to be general for $(u, \bar{H})$, since the block puts more restrictions on the location of the new vertex.

PROPOSITION 4.4 Given a block and hole polyhedron $G(\mathcal{P}, \boldsymbol{p})$, with $\boldsymbol{p}$ in general position for the blocks and holes, then there is an induced gusseted polyhedron $G^{\prime}\left(\mathcal{P}^{\prime}, \boldsymbol{p}^{\prime}\right)$ which is separated and has an isomorphic space of stresses and an isomorphic space of motion assignments.

Proof. List the non-separated vertices of $G$, and for each such vertex, list the blocks and holes around the vertex. We proceed in turn, vertex by vertex, following this arbitrary sequence. As we process a given vertex, we add a gusset to all blocks and holes except the last one in the sequence at the vertex. Given the sequence, the gusseting is unique. As we add new geometric points, we ensure that they are not coplanar with their attachments, and moreover, we ensure that when added they are not collinear with any two of the other vertices of the block or hole. As well, we ensure that they are in general position for the block in one of the swap-equivalent pairs. 
At each stage, the stresses and the motions are isomorphic to the previous polyhedron. By induction and Propositions 4.2, 4.1, we create a final polyhedron and realization with a space of stresses and a space of motion assignments which are isomorphic to the spaces of the original. Since we gusseted all but one of the blocks or holes at a non-separated vertex, this vertex is now separated in the extension, and all vertices added are also separated.

Notice that the two gusseting processes are equivalent, under swapping (Lemma 4.3). We conclude that, provided we use the same sequence of non-separated vertices, and of block and hole faces at these non-separated vertices, we take a pair of swapped block and hole polyhedra to a new pair of separated swapped block and hole polyhedra.

Gusseting is a general 'trick' which can be applied whenever we wish to separate identified polygonal 'holes' or isostatic 'blocks' in polygons. The results for a framework $G(\boldsymbol{p})$, and its gusseted extension $G^{\prime}\left(\boldsymbol{p}^{\prime}\right)$ are local and do not depend on information about the larger scale topological patterns of the framework.

\subsection{Main result revisited}

Using Proposition 4.4 and the techniques of Sections 3.1 and 3.2 we can prove general case versions of Propositions 3.1 and 3.2. Together these results form the proof of the following theorem, which is a general version of Theorem 3.3 .

THEOREM 4.5 Given a block and hole polyhedron, $(\mathcal{P}, \boldsymbol{p})$ with $\boldsymbol{p}$ in general position, there is an isomorphism between the space of motion assignments of the swapped block and hole structure $G^{M}(\overline{\mathcal{P}}, \boldsymbol{p})$ as a hinged panel polyhedron and the space of stresses of the bar and joint framework at the same configuration, $G_{S}(\mathcal{P}, \boldsymbol{p})$.

\section{Proof.}

Combining Proposition 4.4 with Theorem 3.3 and Proposition 2.5 yields the result.

There are some geometric and generic corollaries of these general results. The results can be posed in the contrapositive, since first-order rigidity is equivalent to having only the zero motion assignment, and independence is equivalent to having only the zero stress. This form is appropriate to a number of applications. 


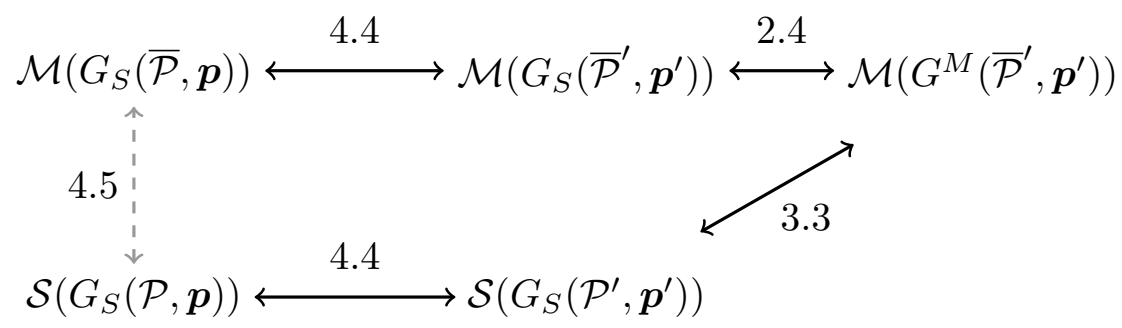

COROLLARY 4.6 Given a block and hole polyhedral framework $G(\mathcal{P}, \boldsymbol{p})$ and the swapped polyhedral framework $G(\overline{\mathcal{P}}, \boldsymbol{p})$,

i) if $G_{S}(\mathcal{P}, \boldsymbol{p})$ is geometrically isostatic then $G_{S}(\mathcal{P}, \boldsymbol{p})$ is geometrically isostatic at the same configuration,

ii) if $G_{S}(\overline{\mathcal{P}}, \boldsymbol{p})$ is geometrically independent then $G^{M}(\mathcal{P}, \boldsymbol{p})$ is geometrically first-order rigid at the same configuration, and

iii) if $G^{M}(\mathcal{P}, \boldsymbol{p})$ is geometrically first-order rigid then $G_{S}(\overline{\mathcal{P}}, \boldsymbol{p})$ is geometrically independent.

Since any generic configuration will also be in general position for all blocks and holes, the geometric results immediately transfer to generic results.

COROLLARY 4.7 Given a block and hole polyhedron $\mathcal{P}$ and the swapped polyhedron $\overline{\mathcal{P}}$,

i) $G^{M}(\mathcal{P})$ is generically rigid if and only if $G_{S}(\overline{\mathcal{P}})$ is generically independent, and

ii) $G_{S}(\mathcal{P})$ is generically isostatic if and only if $G^{M}(\overline{\mathcal{P}})$ is generically isostatic.

There are two special cases which are contained in this general result, and are, implicitly or explicitly, in the prior literature. 


\subsubsection{Example}

An extreme form of the block and hole polyhedron will have no 'surface triangles' and no holes. The polyhedron is composed only of blocks (some of which may happen to be triangles): $\mathcal{P}=\left(\mathcal{B}_{\mathcal{P}}\right)$, while the swapped polyhedron has no blocks: $\overline{\mathcal{P}}=\left(\mathcal{H}_{\overline{\mathcal{P}}}\right)$. In this setting, the key observation is that:

$$
G^{M}(\mathcal{P})=G_{S}(\overline{\mathcal{P}})=G(\mathcal{P})=G(\overline{\mathcal{P}}) .
$$

The entire polyhedron is a body and hinge spherical polyhedron with hinges $G(\mathcal{P})$, and a spherical framwork with edges $G(\overline{\mathcal{P}})=G(\mathcal{P})$ This special form of the result is stated in [3], and is used explicitly in [19] to prove variations on Cauchy's Theorem for convex polyhedra. The correspondence is also implicit (in Euclidean terms) in some remarks in [1].

\subsubsection{Example}

As an intermediate case, we can have only blocks and one hole (no indentified surface triangles): $\mathcal{P}=\left(\mathcal{B}_{\mathcal{P}},\{H\}\right)$. As a hinge structure, this is a disc of rigid panels (blocks), leaving the 'exterior' as a single hole. (See Figure 9.) The swapped structure $\overline{\mathcal{P}}=\left(\{B\}, \mathcal{H}_{\overline{\mathcal{P}}}\right)$ has one block, which we often think of as a rigid ground, and the rest is a bar and joint framework on the edges of the polyhedron. The two connections which are at the core of Section 3 still give an isomorphism between the motion assignments of $G^{M}(\mathcal{P})$ and the self-stresses of $G_{S}(\overline{\mathcal{P}})$. Such 'panel discs' are encountered implicitly in a number of studies such as [18, as well as some recent work on structures built on quadgraphs in discrete differential geometry.

\section{Some Extensions and Further Work}

\subsection{Projective interpretations}

We have used projective coordinates for all of the geometric results in this paper. It is geometrically possible that that some of the 'vertices' or even some of the 'faces' will lie on the plane at infinity in projective space. These actually have valid static and kinematic interpretations, as noted in [3]. For example, a joint at infinity, in our structure, will amount to a set of rigid 


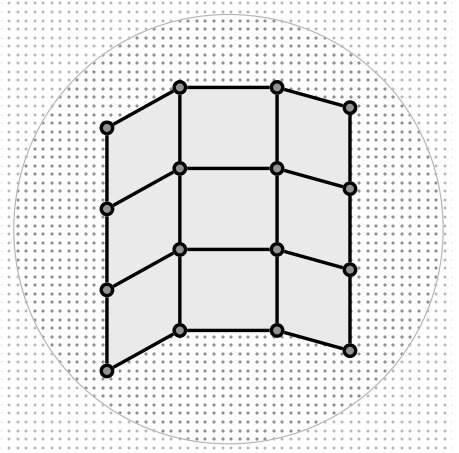

(a)

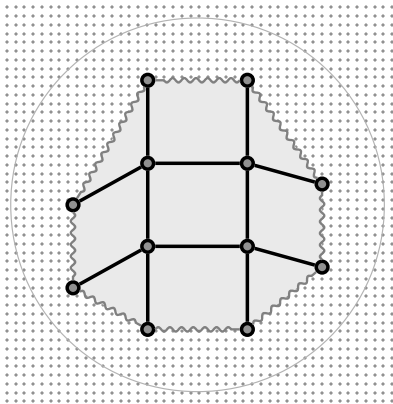

(b)

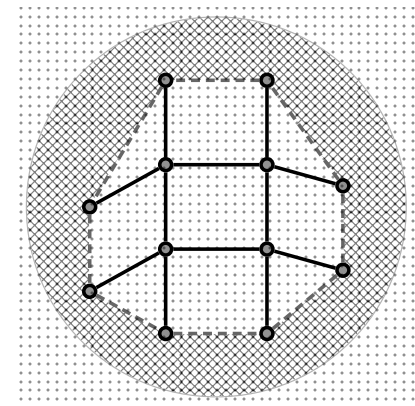

(c)

Figure 9: (a) depicts a block and hole polyhedron $\mathcal{P}$ consisting only of blocks. Figure (b) shows the graph $G^{M}(\mathcal{P})$ (in which two valent vertices have been removed), and (c) depicts the graph $G^{S}(\overline{\mathcal{P}})$ of the swapped polyhedron. This graph can also be viewed as a pinned framework. 
pieces (triangles, blocks) which have hinges which are parallel, pointing to this infinite 'vertex'. A hinge line between two rigid pieces, at infinity, can be represented by a 'slide hinge' between the two panels which leaves only the one degree of freedom between the rigid objects - that is, a translation perpendicular to all the planes through that line at infinity. In this way, using the projective forms includes some additional realizations which actually occur in mechanical engineering.

A second general byproduct of the projective form and invariance of the statics and centers of motion assignments is that all of the geometric (and combinatorial) results work extend to the various metrics which can be extracted from the projective geometry: Euclidean, spherical, and hyperbolic [9].

\subsection{Pure conditions for isostatic block and hole polyhedra}

From earlier work of White and Whiteley [13, we know that any generically isostatic graph $G$ in 3 -space has an associated pure condition $c_{G}$, which is a polynomial in the (projective) coordinates of the vertices. This polynomial represents the special positions in the specific sense that geometrically $G(\boldsymbol{p})$ is isostatic if and only $c_{G}(\boldsymbol{p}) \neq 0$. The polynomial is defined up to multiplication by a non-zero scalar.

It was also shown that an isostatic component $G^{*}$ of $G$, with at least four vertices, creates a polynomial factor of the pure condition $c_{G}$ which is the pure condition $c_{G^{*}}$ of its subframework. So the pure condition of an isostatic block and hole polyhedron $\mathcal{P}$ would have the form

$$
c_{G(\mathcal{P})}=T(\mathcal{P}) \prod_{B_{i} \in \mathcal{B}_{\mathcal{P}}}\left(c_{B_{i}}\right)
$$

for some surface polynomial $T(\mathcal{P})$ which does not depend on the specific isostatic framework inserted into the blocks.

Similarly, for the swapped block and hole polyhedron $\overline{\mathcal{P}}$, which is also isostatic, we have

$$
c_{G(\overline{\mathcal{P}})}=T(\overline{\mathcal{P}}) \prod_{H_{i} \in \mathcal{H}_{\overline{\mathcal{P}}}}\left(c_{H_{i}}\right)
$$

where $c_{H_{i}}$ represents the pure condition of the isostatic framework inserted into the dual blocks $\bar{B}_{i}=H_{i}$.

If we happen to change the isostatic subframework for the block $B_{i}$, its factor will change, but $T(\overline{\mathcal{P}})$ will not, even if we add additional vertices inside the block. 


\subsubsection{Example}

Consider the elementary example $\mathcal{C}$ in Figure 2a, in which the block is labelled $v_{1} v_{2} v_{3} v_{4}$ and the hole is labeled $v_{5} v_{6} v_{7} v_{8}$. For simplicity in the following formulas, we re-label the hole vertices as follows: $v_{5}=u_{1}, v_{6}=$ $u_{2}, v_{7}=u_{3}, v_{8}=u_{4}$. Writing $[\boldsymbol{a b} \boldsymbol{c d}]$ for the determinant of the $4 \times 4$ matrix of projective coordinates of the four points $\boldsymbol{a}, \boldsymbol{b}, \boldsymbol{c}, \boldsymbol{d}$, the pure condition has the form:

$$
c_{\mathcal{C}}=\left[\boldsymbol{v}_{\mathbf{1}} \boldsymbol{v}_{\mathbf{2}} \boldsymbol{v}_{\mathbf{3}} \boldsymbol{v}_{\mathbf{4}}\right]\left(\prod_{i=1 . .4}\left[v_{i} v_{i+1} u_{i} u_{i+1}\right]-\prod_{i=1 . .4}\left[v_{i} v_{i-1} u_{i} u_{i+1}\right]\right)
$$

By convention, we are cycling the indexes so $v_{i+1}=v_{1}$ when $i=4$. Notice the factor $\left[v_{1} v_{2} v_{3} v_{4}\right]$, for that block, which says that the complete graph $K_{4}$ is isostatic unless the four points are coplanar. If we consider the swapped block and hole polyhedron, with the block at $v_{5} v_{6} v_{7} v_{8}$, then (by symmetry)

$$
c_{\overline{\mathcal{C}}}=\left[\boldsymbol{v}_{\mathbf{5}} \boldsymbol{v}_{\mathbf{6}} \boldsymbol{a}_{\mathbf{7}} \boldsymbol{v}_{\mathbf{8}}\right]\left(\prod_{i=1 . .4}\left[v_{i} v_{i+1} u_{i} u_{i+1}\right]-\prod_{i=1 . .4}\left[v_{i} v_{i-1} u_{i} u_{i+1}\right]\right)
$$

In particular, we see $T(\mathcal{C})=T(\overline{\mathcal{C}})$.

We believe this is typical of conditions for isostatic block and hole polyhedra and their swapped polyhedra. ¿From our results here, we do know that any configuration $\boldsymbol{p}$ for which $(\mathcal{P}, \boldsymbol{p})$ has a self-stress will also provide a self stress on $(\overline{\mathcal{P}}, \boldsymbol{p})$ a self-stress. Equivalently, $T(\mathcal{P}, \boldsymbol{p})=0$ if and only if $T(\overline{\mathcal{P}}, \boldsymbol{p})=0$, over all configurations $\boldsymbol{p}$ of points in the real projective space. We have a stronger conjecture which is compatable with this observation.

CONJECTURE 5.1 Given a generically isostatic block and hole polyhedron $\mathcal{P}$, the surface polynomial of $\mathcal{P}$ is the same as the surface polynomial of the swapped polyhedron $T(\mathcal{P})=T(\overline{\mathcal{P}})$

Even adding a gusset to $\mathcal{P}$ to create $\mathcal{P}^{\prime}$ will show up as factoring in these pure conditions. The 3 -valent insertion at a hole of $\mathcal{P}$, (or onto the modified block in $\mathcal{P}^{\prime}$ ) leaves the residual framework isostatic, so this gusset creates a small factor $\left[u_{i-1} u_{i} u_{i+1} v_{i}\right]$ where $u_{i-1} u_{i} u_{i+1} v_{i}$ are the four points that make up the gusset. When we compare the surface polynomials of $\mathcal{P}$ and $\mathcal{P}^{\prime}$, we see that $T\left(\mathcal{P}^{\prime}\right)=\left[\boldsymbol{u}_{\boldsymbol{i}-\mathbf{1}} \boldsymbol{u}_{\boldsymbol{i}} \boldsymbol{u}_{\boldsymbol{i}+\mathbf{1}} \boldsymbol{v}_{\boldsymbol{u}_{\boldsymbol{i}}}\right] T(\mathcal{P})$ if the gusset was added at a hole. For a block gusset, one has this factor as well an additional substitution of $v_{i}$ in place of $u_{i}$, in the block polynomial factor. In particular, these steps make the same change to the surface polynomials $T(\mathcal{P})$ and $T(\overline{\mathcal{P}})$. That is, 
they will be the same after the addition of a gusset if and only if they were the same polynomial before.

More generally, we anticipate that the pure conditions of block and hole polyhedra will have nice algebraic geometric forms which would be interesting to explore. For example, if a block and hole polyhedron has four quadrilateral faces $F^{1}, F^{2}, F^{3}, F^{4}$ which are blocks or holes, and if all choices of two of these for blocks and two of these for holes generated a generically isostatic block and hole polyhedron, then we can ask whether there is a single 'surface condition' which is shared by all six possible choices? This type of question is a subject for further research.

\subsection{More general spheres}

So far we have assumed the combinatorial structure was a 3-connected sphere. The actual proofs do not require the 3-connectivity, just the topology of a spherical polyhedron. The results would work perfectly well for 2-connected planar graphs which have identified holes, blocks and 'surface faces'. One point requiring some care for 2-connected polyhedra is that when the surface faces are triangulated, we do not accidently insert the same pair of vertices as an 'edge' in two different faces, making $G^{M}(\mathcal{P})$ a multi-graph. In order not to revert to a 3-connected graph when doing this triangulation, some of the faces at the 2-disconnection will need to be holes or blocks. On the other hand, by inserting gussets into blocks and holes, one can convert to structure into an equivalent (for statics and kinematics) 3-connected sphere.

A second point is the possibility of two faces sharing two edges. If these are both blocks, we will have two dual hinges joining the two blocks, so $G^{M}(\mathcal{P})$ is no longer a graph and the two blocks are locked as one rigid object.

Under these small changes, the results will generalize to block and hole polyhedra on 2-connected spheres.

\subsection{Surfaces with other toplogies}

All of these results were written with the assumption that we started with a spherical polyhedron. The key to these proofs is actually broader than just the spherical polyhedral topology.

In Proposition 3.2 , the proof from the motion assignment to the selfstress relied centrally on the fact that at each edge there were two faces of the original structure (including the blocks and holes) and that at each 'interior' vertex there was a face-edge cycle, as well as a cycle surrounding each hole- 
face. These conditions are closely related to the definition of an oriented surface as a complex of faces, edges and vertices. They are also sufficient to generate a self-stress from the motion assignment. So Proposition 3.2 extends to generalized block and hole polyhedra on arbitrary closed oriented polyhedral surfaces.

If we reread the proof of Proposition 3.2, it becomes clear that what was used was that there was an underlying surface (faces, vertices, edges) and that each vertex was surrounded by an face-edge cycle. So the result transfers to surfaces of any genus. A motion assignment on a generalized block and hole surface becomes a self-stress of the swapped structure as a bar and joint framework.

The extensions using gussets (Propositions 4.14.2 and Lemma 4.3) also apply to these generalized block and hole polyhedra, giving isomorphic spaces before and after adding gussets both for stresses and for motions. This extends the transformation to generalized block and hole polyhedra on closed oriented polyhedral surfaces.

For other oriented surfaces beyond the sphere, the converse Proposition 3.1 does not extend. For example, a triangulated torus has $|E|=3|V|$, and the are always at least a six dimensional space of self-stresses, including for the space of generic realizations which are first-order rigid. For a torus, any vertex-face cycle which is not homologous to zero will not be guaranteed to have a zero sum in the self-stress, since it is not a cut set. However, in a motion assignment, this sum on this cycle must be zero. In a general torus, there are two such cycles which are not homologous, and these generate all the cycles. If the sum of the self-stress around each of these cycles is also zero, then the self-stress will induce a motion assignment. This cycle subspace of self-stresses is isomorphic to the space of motion assignments. Moving to block and hole polyhedra simply extends this problem with some additional complexity.

\subsection{Polarity and swapping}

There is a theory which explores the rigidity of polar structures for bar and joint structures in 3-space, also called sheet structures [16]. In this transformation, plane-isostatic faces (including triangles) go to vertices, edges go to edges and vertices go to plane isostatic frameworks on the plane containing the polar edges (also called sheets). The net result is a polar structure which can be realized as a bar and joint framework. This is a polar transformation which applies to arbitrary bar and joint frameworks in 3-space, preserving static and infinitesimal rigidity (and therefore taking isostatic frameworks 
to isostatic sheet structures). This is a geometric transformation, not just a combinatorial process.

We will not give the details here, but this polarity has a direct application to block and hole polyhedra and the swapping we have examined here. When this polarity is applied to a polyhedron with isostatic faces, it produces the dual polyhedron with isostatic faces [16]. When the polarity is applied to a rigid block, it produces a dual rigid block. Overall, when the polarity is applied to a block and hole polyhedron, it will produce a dual block and hole polyhedron. When it is applied to the swapped polyhedron, it will produce the swapped polyhedron of the dual block and hole polyhedron.

\section{References}

[1] A.D. Alexandrov: Convex polyhedra, GTI, Moscow, 1950. English translation: Springer, Berlin, 2005.

[2] A.L. Cauchy: A.L. Cauchy, Recherche sur les polydres - premier mémoire, Journal de l'Ecole Polytechnique 9 (1813), 6686.

[3] Henry Crapo and Walter Whiteley: Statics of frameworks and motions of panel structures: a projective geometric introduction, Structural Topology 1 (1982), pp 43-82.

[4] M. Dehn: Über die Starreit konvexer Polyeder, (in German), Math. Ann. 77 (1916), 466-473.

[5] Wendy Finbow-Singh and Walter Whiteley: Isostatic Almost Spherical Frameworks via Disc Decomposition, preprint, York University, 2007

[6] Herman Gluck: Almost all simply connected closed surfaces are rigid Lecture Notes in Math. 438, Geometric Topology, Springer-Verlag (1975), 225-239.

[7] JaCK Graver: Counting on Frameworks Dolciani Mathematical Expositions, MAA, Washington. 2001.

[8] Jack Graver, Brigitte Servatius, and Hermann Servatius: Combinatorial Rigidity Graduate Studies in Mathematics, AMS, Providence. 1993. 
[9] Franco Saliola and Walter Whiteley: Equivalence of Firstorder Rigidity for Euclidean, Spherical and Hyperbolic Metrics, Preprint, York University, 2004.

[10] Tiong-Seng Tay and Walter Whiteley: Generating isostatic frameworks, Structural Topology 11 (1985), pp 20-69.

[11] N. White: Geometric applications of the Grassmann-Cayley algebra, Chapter 59 in Handbook of Discrete and Computational Geometry, J. Goodman and J. O'Rourke (eds), CRC Press LLC, Boca Raton, FL; Second Edition, (2004).

[12] N. White: A tutorial on Grassmann-Cayley algebra In Invariant Methods in Discrete and Computational Geometry, N. White, Ed. Kluwer Academic Publisher, (1995), pp. 93-106.

[13] Neil L. White And Walter Whiteley: The Algebraic Geometry of Stresses in Frameworks. SIAM Journal on Algebraic and Discrete Methods 4 (1983), 481-511.

[14] W. Whiteley: Rigidity and Scene Analysis; in Handbook of Discrete and Computational Geometry, J. Goodman and J. O'Rourke (eds.), (second edition) (2004), 1327-1354.

[15] W. Whiteley: Matroids from discrete geometry, in Matroid Theory, J. E. Bonin, J. G. Oxley, and B. Servatius, Eds. American Mathematical Society, Contemporary Mathematics, 1996, vol. 197, pp 171313.

[16] W. Whiteley: Rigidity and polarity I: statics of sheetworks; Geometriae Dedicata 22 (1987), 329-362

[17] W. Whiteley: Infinitesimally rigid polyhedra II: modified spherical frameowrks, Trans. A.M.S. 306 (1988), 115-139.

[18] W. Whiteley: Infinitesimal motions of a bipartite framework Pac. J. Math. 110 (1984), 233-255.

[19] W. Whiteley: Infinitesimally rigid polyhedra I: statics of frameworks, Trans. A.M.S., 285 (1984), 431-465. 